\title{
Atypical Lymphocyte to Leukocyte Ratio
}

National Cancer Institute

\section{Source}

National Cancer Institute. Atypical Lymphocyte to Leukocyte Ratio. NCI Thesaurus. Code C64819.

The determination of the ratio of atypical lymphocytes to leukocytes in a blood sample.

The measurement may be expressed as either a ratio or a percentage. 